\title{
Frontières
}

\section{Le cadavre dans la littérature fantastique}

\section{La place du corps dans Frankenstein de M. Shelley et Le Rêve du docteur Mišić de K.S. Gjalski}

\section{Isabelle Percebois}

Volume 23, numéro 2, printemps 2011

Enquêtes sur le cadavre : 2. Fantastique

URI : https://id.erudit.org/iderudit/1007583ar

DOI : https://doi.org/10.7202/1007583ar

Aller au sommaire du numéro

\section{Éditeur(s)}

Université du Québec à Montréal

ISSN

1916-0976 (numérique)

Découvrir la revue

Citer cet article

Percebois, I. (2011). Le cadavre dans la littérature fantastique : la place du corps dans Frankenstein de M. Shelley et Le Rêve du docteur Mišić de K.S. Gjalski. Frontières, 23(2), 7-13. https://doi.org/10.7202/1007583ar

\section{Résumé de l'article}

$\mathrm{Au} \mathrm{XIX}{ }^{\mathrm{e}}$ siècle, la littérature fantastique exploite le thème de la mort et devient une véritable littérature du cadavre. Dans des oeuvres telles que Frankenstein de Mary Shelley ou Le Rêve du docteur Mišić de K.S. Gjalski, les corps errent entre la vie et la mort et s'animent sous la plume des écrivains. Ces récits substituent le cadavre au traditionnel revenant et délaissent le surnaturel pour puiser l'horreur dans la réalité : le corps inerte y apparaît comme un objet de recherches scientifiques et fait du cimetière l'antichambre du laboratoire. À travers l'histoire du docteur Frankenstein et du docteur Mišić, le lecteur découvre la face sombre d'un siècle scientiste où la dépouille, devenue simple marchandise, se négocie et s'expose dans les salles de dissections. Les amphithéâtres des universités font ainsi de la mort un spectacle où les savants se prennent pour Dieu ; mais en défiant l'interdit et en brisant le tabou entourant le cadavre pour dévoiler les secrets de la nature, les héros fantastiques encourent un châtiment mortel. 


\section{Résumé}

Au XIX siècle, la littérature fantastique exploite le thème de la mort et devient une véritable littérature du cadavre. Dans des œuvres telles que Frankenstein de Mary Shelley ou Le Rêve du docteur Mišić de K.S. Gjalski, les corps errent entre la vie et la mort et s'animent sous la plume des écrivains. Ces récits substituent le cadavre au traditionnel revenant et délaissent le surnaturel pour puiser l'horreur dans la réalité: le corps inerte y apparaît comme un objet de recherches scientifiques et fait du cimetière l'antichambre du laboratoire. À travers I'histoire du docteur Frankenstein et du docteur Mišić, le lecteur découvre la face sombre d'un siècle scientiste où la dépouille, devenue simple marchandise, se négocie et s'expose dans les salles de dissections. Les amphithéâtres des universités font ainsi de la mort un spectacle où les savants se prennent pour Dieu; mais en défiant I'interdit et en brisant le tabou entourant le cadavre pour dévoiler les secrets de la nature, les héros fantastiques encourent un châtiment mortel.

Mots clés: fantastique-cadavrescience-médecine-tabou.

\section{Abstract}

In the 19th century, fantastic literature draws on the theme of death and becomes a true literature of the corpse. In works such as Mary Shelley's Frankenstein or K.S. Gjalski's Le Rêve du docteur Mišić, bodies wander between life and death and come to life under the writer's pen. These narratives substitute the corpse for the traditional ghost and abandon the supernatural to draw horror from reality: the inert body there appears as a subject of scientific research so that the churchyard is a necessary stop on the way to the laboratory. Through the stories of Dr. Frankenstein and Dr. Mišić, the reader discovers the dark face of a scientist century where the body, turned into mere merchandise, can be bought and exhibited in dissection rooms. Thus university amphitheaters turn death into a show in which scientists play God; but by defying conventions and breaking the taboo surrounding corpses to unveil the secrets of nature, fantastic heroes incur a deadly punishment.

Keywords: fantastique-corpsescience-medicine-taboo.

\section{LE CADAVRE DANS LA LITTÉRATURE} FANTASTIQUE La place du corps dans Frankenstein de M. Shelley et
Le Rêve du docteur Mišić
de K.S. Gjalski

\author{
Isabelle Percebois, \\ professeure agrégée de lettres modernes, \\ doctorante en littérature comparée, \\ Université Paris-IV Sorbonne.
}

La mort constitue l'un des thèmes essentiels de la littérature fantastique, qui se nourrit des peurs les plus primaires de l'homme. Elle incarne le mystère de la condition humaine et apparaît comme l'image même de l'inatteignable, de l'indicible et de l'inquiétant. Ouvrant la porte au surnaturel, elle inspire les auteurs qui transforment cette mort en un véritable univers peuplé de monstres sanguinaires et d'âmes errantes. Dans les œuvres $\mathrm{du} \mathrm{XIX}^{\mathrm{e}}$ siècle, les vampires et les revenants se révèlent ainsi de nouveaux héros et invitent les lecteurs à «entrer dans la mort». Mais si le fantastique est bien une littérature de la mort, il apparaît également comme une littérature du cadavre, tant le corps prend d'importance dans les récits de l'époque. Les chefs-d'œuvre du genre, tels que Frankenstein (1818) de Mary Shelley, témoignent de cette évolution; toute la littérature fantastique européenne en porte la marque, à l'image du texte de Ksaver Šandor Gjalski ${ }^{1}$ intitulé Le Rêve du docteur Mišić, qui reste moins connu du grand public. Loin de réduire les cadavres à de simples dépouilles, ces œuvres mettent en scène des corps ramenés à la vie et soulignent la fascination exercée par le cadavre: "fascination de la confrontation à l'inatteignable et répulsion face à ce qui se décompose» (Debout et Cettour, 2006, p. 62). Alors que les facultés de médecine des universités se changent en décors du fantastique, l'on assiste ainsi à la théâtralisation du corps, devenu objet de sciences et de connaissances. Enfin, dans ces œuvres, le cadavre apparaît également comme l'enjeu d'une lutte entre science et religion: en brisant le tabou qui entourait le corps, 
le personnage du savant croyait trouver la clef pour résoudre le mystère de la mort et prendre la place de Dieu.

\section{ANIMER LE CORPS INERTE}

Dans la littérature fantastique, le cadavre n'est pas simplement le "corps d'un être humain qui a cessé de vivre», selon la définition proposée par le Trésor de la Langue Française, mais un corps souvent ranimé, et rappelé à la vie. Défiant les règles de la nature, il dépasse les frontières de cette définition et offre une représentation inédite de la mort dans la littérature: au travers de ce corps, les auteurs fantastiques explorent les abîmes de l'horreur et prolongent ainsi l'œuvre du courant gothique. D'ordinaire habité par un esprit vengeur ou quelque démon, le cadavre apparaît dans les récits à mi-chemin entre le mort-vivant et le fantôme, deux grands classiques du fantastique. Dans l'œuvre de Gjalski intitulée Le Rêve du docteur Mišić, le héros éponyme est un jeune médecin à l'esprit cartésien qui voit sa vie bouleversée par des rêves étranges et prémonitoires, de plus en plus réalistes au fil des pages, où apparaît une jeune et belle bohémienne, au corps froid comme la mort. Le corps de la jeune femme qui hante le héros semble ainsi fuyant, illusoire comme une apparition, et néanmoins terriblement réel entre les mains du personnage. On remarque que c'est au moment où le docteur Mišić doute de la réalité et croit rêver éveillé que ce corps surgit devant ses yeux, éblouissant de charme et de beauté :

Sur le sol en pierre gisait le corps d'une jeune fille. On ne pouvait distinguer si elle était morte ou si elle reposait. Toutefois, il avait la sensation que les yeux de la jeune fille le fixaient. [...] Ces yeux qui le regardaient étaient merveilleux, immenses et pleins de charme, le regard était calme et profond (Gjalski, 1996, p. 404-405).

La jeune fille décrite dans ces quelques lignes n'a rien de l'image traditionnelle du mort-vivant imposée par la littérature et le cinéma de notre époque, qui se rapproche davantage du zombie tel qu'il apparaît dans La Nuit des morts-vivants de George A. Romero (1968), ou dans les productions plus récentes qui s'en inspirent, comme 28 jours plus tard de Danny Boyle (2002). Il n'est nullement question de chair en décomposition chez Gjalski, qui célèbre tout au long de la nouvelle la beauté surnaturelle de la bohémienne. Dans cet extrait, le docteur Mišić semble même manquer de qualificatifs pour décrire le regard de la jeune femme ensorcelante qui s'est emparée de son esprit. Et si le héros apparaît lui-même comme un être égaré entre la veille et le sommeil, c'est entre la vie et la mort qu'évolue ce "charmant» cadavre, aux caresses envoûtantes. En cela, la jeune bohémienne du récit de Gjalski s'apparente à la "morte amoureuse » créée par Théophile Gautier en 1836. Les ressemblances sont nombreuses entre ces deux œuvres puisque le narrateur de La Morte amoureuse, le prêtre Romuald, est également une âme perdue entre le rêve et la réalité: "et lorsqu'au lever de l'aube je me réveillais, il me semblait au contraire que je m'endormais et que je rêvais que j'étais prêtre»(Gautier, 2004, p. 26). De même, l'on découvre que la dame de ses pensées, la belle Clarimonde, n'est autre qu'une femme vampire se nourrissant chaque nuit de son sang et prolongeant dans ses cauchemars son existence démoniaque. En référence à cette œuvre fantastique, Gjalski suggère que la jeune bohémienne pourrait, elle aussi, se délecter du sang humain et préserver sa beauté grâce à ses maléfices: "Ses baisers étaient glacés-il croyait en perdre tout son sang. Pourtant il continua à l'embrasser, sombrant lentement dans la torpeur» (Gjalski, 1996, p. 405). Toutefois, l'auteur ne souhaite pas céder totalement à la mode du vampirisme qui traverse le $\mathrm{XIX}^{\mathrm{e}}$ siècle, depuis Le Vampire (1819) de John W. Polidori jusqu'au célèbre Dracula (1897) de Bram Stoker, et abandonne bien vite cette explication simpliste.

En effet, le personnage de la jeune bohémienne s'inspire davantage d'œuvres littéraires empruntées au romantisme noir, à la mythologie ou au folklore russe. Comme les roussalki des légendes slaves, ces créatures sublimes qui charmaient les hommes pour mieux les étouffer sous leurs caresses, elle entraîne le docteur Mišić dans la mort en le couvrant de baisers; mais sa beauté ensorcelante évoque également les succubes de la Grèce antique, qui utilisaient les charmes de leur corps glacé pour séduire les hommes jusque dans leur sommeil. Néanmoins, dans la mythologie, les succubes sont décrits comme des esprits, et il n'existe aucun être, bienfaisant ou démoniaque, qui puisse donner un nom au cadavre de la jeune bohémienne. En outre, il est intéressant de noter que cette jeune fille n'a pas de nom dans le récit car l'auteur veut souligner que la mort lui a ôté toute possibilité «d'être», et qu'elle n'est plus qu'un corps errant dans les limbes, qu'un « objet-chair ${ }^{2}$ »dépourvu d'identité. Pour décrire ce personnage, l'on pourrait citer les mots de François Mauriac, déclarant dans son Journal qu'un «cadavre est essentiellement une absence, une chose quittée, rejetée-une dépouille enfin. Dans l'affreux désarroi que nous éprouvons au spectacle d'une mort, il entre un sentiment de duperie: celui que nous aimons est là et il n'est plus là » (Mauriac, 1970, p. 38). C'est en parcourant les cimetières et en assistant aux cérémonies du «jour des Morts» que l'écrivain s'abandonne à ces réflexions, qui trouvent un écho dans la littérature fantastique. Certes, comme l'explique Mauriac, le cadavre peut nous «dupe[r] » en nous laissant croire que l'être disparu est toujours présent; mais cette tromperie est d'autant plus grande dans les textes fantastiques que le cadavre est bel et bien présent, capable de se mouvoir et de provoquer la peur.

Dans Frankenstein, en revanche, on ne peut parler «d'un » cadavre réanimé dans la mesure où la créature monstrueuse est formée d'un assemblage de corps dérobés aux cimetières et aux charniers: "After having formed this determination and having spent some months in successfully collecting and arranging my materials, $I$ began» (Shelley, 1992, p. 54) ${ }^{3}$. Le monstre qui prend vie entre les mains du docteur Frankenstein n'est donc pas l'image déformée d'une personne disparue, mais un être appartenant à une nouvelle espèce, une «œuvre» artistique aux yeux du savant enthousiaste. Dans la première partie du roman, le créateur se plaît à rêver à la reconnaissance que lui exprimerait cet être doué de vie, et en vient même à oublier l'aspect monstrueux de cet amas de chairs dérobées aux corps en décomposition. Ce n'est qu'après sa "naissance» et ses premiers crimes que le docteur Frankenstein emploie le terme de «monstre» pour désigner la créature dans son récit des événements. Alors que la jeune bohémienne brillait par sa beauté sans pareille dans la nouvelle de Gjalski, c'est la laideur qui caractérise le monstre et le pousse à fuir la compagnie des hommes dans le roman de Mary Shelley. Son aspect hideux est évoqué à de nombreuses reprises, et l'horreur exprimée par le docteur Frankenstein n'a d'égal que le dégoût ressenti par la créature elle-même: "God, in pity, made man beautiful and alluring, after his own image; but my form is a filthy type of yours, more horrid even from the very ressemblance»(Shelley, 1992, p. 133) ${ }^{4}$. Dans cet extrait, Mary Shelley réécrit un passage de la Genèse puisque, contrairement à Adam, cet être, qui est l'image de la mort elle-même, ne peut se reconnaître dans son créateur. Condamné à la solitude de sa condition, il erre lui aussi dans un monde qui n'est pas le sien, emprisonné entre la vie et la mort dans un purgatoire terrestre. Et si son corps hideux est bien doué de vie et d'une force prodigieuse, il n'est en rien "vivant» et évoque les mots de Michel Debout dans Science et mythologie du mort : "La machine ne meurt pas, 
elle n'a pas d'histoire, tout au plus elle cesse de fonctionner. Le corps "biologisé" à l'aune du modèle de la machine ne meurt pas tout à fait parce qu'il ne vit pas tout à fait!» (Debout et Cettour, 2006, p. 166.) La créature de Frankenstein, dénuée de nom et de vie, apparaît donc comme une machine humaine ou un cadavre animé, et ne saurait atteindre la dignité de l'être humain.

Dans l'œuvre de Mary Shelley, ce sont les pouvoirs de la science qui parviennent à réanimer les cadavres, et notamment les vertus extraordinaires de l'électricité. L'on sait que l'auteur s'est grandement inspiré des travaux de Luigi Galvani et de ses successeurs, au XIX ${ }^{\mathrm{e}}$ siècle, pour donner de la vraisemblance à son récit comme à son héros. C'est pourquoi les premiers chapitres de l'œuvre sont consacrés aux années de formation du jeune Frankenstein, découvrant sa passion pour l'anatomie et les sciences exactes à l'université d'Ingolstadt. Alors que, dans Le Rêve du docteur Mišić, la magie du rêve parvenait à rendre la vie au corps inerte, c'est la fée électricité qui anime-ou plutôt réanime-la créature cadavérique dans Frankenstein: "With an anxiety that almost amounted to agony, I collected the instruments of life around me, that I might infuse a spark of being into the lifeless thing that lay at my feet » (Shelley, 1992 , p. 58 $)^{5}$. Bien entendu, la description du procédé scientifique reste très vague, voire métaphorique avec l'évocation de cette "étincelle» animant soudain l'amas de chair inerte. En évoquant un procédé électrique, elle renforce la vraisemblance du récit et constitue un effet de réel destiné à persuader le lecteur de la véracité des faits. Ainsi, l'épisode de la «naissance» de la créature présente un grand intérêt dans notre réflexion sur le cadavre; en effet, l'électricité parvient à ranimer ce corps-ou plutôt ce monceau de corps-de manière quasi magique et nous amène à nous interroger sur cette «vie» rendue au cadavre. En latin, le mot d'anima est polysémique, désignant aussi bien la vie en général que l'air que l'on respire; si l'on considère ces acceptions du terme, Frankenstein anime réellement la créature puisqu'il décrit en détail son premier souffle et «sa respiration pénible». Sa découverte révolutionnaire s'apparente donc au concept du «souffle vital», ou pneuma, développé dans la philosophie grecque. Mais n'oublions pas qu'en latin anima signifie également l'âme, en tant que principe spirituel : l'on peut donc se demander si le savant parvient à créer un être doué d'une âme véritable. Cette interrogation, qui sous-tend l'ensemble du roman, est essentielle car sans âme, la créature n'est qu'un cadavre animé, tandis que l'âme lui conférerait une dignité d'être humain. Mary Shelley ne tranche pas la question dans le roman, et laisse au lecteur le choix de l'interprétation. Au regard des crimes odieux et des nombreux meurtres commis par le monstre, on est tenté de suivre l'opinion du docteur Frankenstein et de voir dans cette créature un simple démon enfanté pour répandre le mal et la destruction; toutefois, ses larmes et son attitude compatissante envers son créateur, à la fin du roman, pourraient aisément suggérer le contraire. En abordant la problématique du cadavre, Mary Shelley soulève donc des questions aussi bien religieuses, existentielles que scientifiques.

\section{LE CADAVRE, OBJET DE SCIENCE ET DE CONNAISSANCE}

Dès le début du XIX ${ }^{\mathrm{e}}$ siècle, le cadavre, le corps en décomposition, tend à remplacer dans les textes fantastiques le revenant cher aux récits gothiques, comme si l'horreur n'était plus à chercher dans le surnaturel mais dans la déchéance même de la nature. Jean-Jacques Lecercle résume ce phénomène dans son étude consacrée à l'œuvre de Mary Shelley en soulignant que «le fantastique n'a pas besoin du surnaturel-il se contente le plus souvent de la réalité quotidienne» (Lecercle, 1990, p. 68). Il faut garder à l'esprit que le cadavre n'est pas considéré comme un être à part entière : privé de l'âme qu'il abritait, il n'est plus qu'une coquille vide, qu'un objet livré aux mains des savants. Cette réification est la condition même de la recherche scientifique qui ne peut étudier un corps sans faire abstraction de tout sentimentalisme: «Il y a deux façons de se comporter dans cette confrontation aux cadavres: la première-purement défensive-consiste à "déshumaniser" le corps, le réduire à un cesse pour désigner les cadavres que le savant exhume dans les cimetières pour les besoins de ses expériences, et bâtir sa créature monstrueuse: "I collected bones from charnel-houses and disturbed, with profane fingers, the tremendous secrets of the human frame. [...] The dissecting room and the slaughter-house furnished many of my materials» (Shelley, 1992, p. 55$)^{6}$. Cette idée du corps-objet est un emprunt de la littérature à la science du $\mathrm{XIX}^{\mathrm{e}}$ siècle, dont les progrès passent par la compréhension de la nature, et de la nature humaine notamment. C'est l'époque de la révolution médicale, de l'essor de la chirurgie et des sciences de l'homme, et l'engouement est tel que le cadavre devient même une "denrée recherchée», selon les mots d'Anne Carol (2004, p. 256). Dans ce siècle scientifique, les universités de médecine et les salles de dissection s'arrachent même ces corps censés détenir la clef du mystère de la Création. C'est pourquoi le cimetière devient l'antichambre du laboratoire du docteur Frankenstein qui y réalise ses expériences et cherche à y percer le secret de la vie. Dans les premiers chapitres du roman, Mary Shelley s'attarde même davantage sur ses recherches au cœur de la mort que sur son ultime découverte résumée en ces termes: "a sudden light broke in upon me»(Shelley, 1992, p. 53) ${ }^{7}$. Elle entraîne son lecteur jusqu'au fond des caveaux pour admirer l'œuvre de la mort et la lente liquéfaction des cadavres:

My attention was fixed upon every object the most insupportable to the delicacy of the human feelings. I saw how the fine form of man was degraded and wasted; I beheld the corruption of death succeed to the blooming cheek of life; I saw how the worm inherited the wonders of the eye and brain (Shelley, 1992, p. 53) ${ }^{8}$.

DÈS LE DÉBUT DU XIX SIÈCLE, LE CADAVRE, LE CORPS EN DÉCOMPOSITION, TEND À REMPLACER DANS LES TEXTES FANTASTIQUES LE REVENANT CHER AUX RÉCITS GOTHIQUES, COMME SI L'HORREUR N'ÉTAIT PLUS À CHERCHER DANS

LE SURNATUREL MAIS DANS LA DÉCHÉANCE MÊME DE LA NATURE.

objet » (Debout et Cettour, 2006, p. 93). Il n'est pas question de travail de deuil dans le monde de la science car le corps devient un simple «objet », un laboratoire humain ouvrant un champ de recherches extraordinaire. Dans Frankenstein, on peut noter que le terme de «matériaux» revient sans
Le docteur Frankenstein se plaît à contempler les ravages irréparables de la mort et n'épargne au lecteur aucun détail morbide, pas même le travail des vers rongeant les tissus humains. Dans son récit, le cimetière n'a plus aucune dimension sacrée et se présente comme un terrain 
d'observation pour le scientifique avide de connaissances. Mary Shelley rompt totalement avec les courants gothique et romantique qui faisaient de ce lieu le cadre de cérémonies mystiques ou magiques, comme chez Matthew G. Lewis notamment: "Le cimetière n'est pas ici le lieu où nécromants et spagiristes s'adonnent à la magie noire et se livrent à des recherches sacrilèges; c'est, pour l'étudiant Frankenstein, comme un vaste laboratoire servant à une recherche scientifique et non à la sorcellerie»(Palacio, 1969, p. 100). On peut également remarquer que, dans cette partie du roman, le savant s'enferme peu à peu dans la solitude de sa quête de connaissances. Loin de sa famille et de sa tendre Elizabeth, le docteur Frankenstein fait abstraction du monde qui l'entoure et rejette la compagnie des hommes au profit de celle des cadavres. La recherche scientifique et ses enjeux lui imposent ce sacrifice volontaire et excluent de son existence les sentiments d'amitié et d'amour. Aux yeux du héros, cette mort sociale n'est rien car seul importe l'avenir de la science; pour percer les secrets de la nature, il se révèle prêt à explorer le mystère du corps humain et à étudier les cadavres comme de simples corps-objets.

Matériau scientifique, le cadavre est même réduit au rang de "marchandise» dans le texte de Gjalski où il est question d'un véritable commerce des corps. $\mathrm{Au}$ cours d'un rêve, le docteur Mišić se retrouve ainsi dans un amphithéâtre de la Faculté de médecine de Vienne, surgi de ses souvenirs d'étudiant. Il est à nouveau confronté au corps inerte de la belle bohémienne, l'objet de ses désirs et de sa tentation, qui semble offert aux yeux du spectateur et rappelle les morgues «spectacles» étudiées par Bruno Bertherat (Bertherat, 2005, p. 181). Cet épisode du rêve illustre l'obsession grandissante du héros mais nous fait également découvrir le sombre visage des universités de médecine du XIX ${ }^{\mathrm{e}}$ siècle: "Cette fois, il était étudiant, à la faculté de Vienne, et se trouvait dans la salle d'anatomie. L'huissier lui disait qu'il lui avait procuré un corps entier à disséquer, qu'il s'était donné beaucoup de mal pour l'avoir et que pour cette raison tous les autres lui en voulaient» (Gjalski, 1996, p. 410). L'auteur fait référence dans ce récit à une réalité historique puisque l'on sait que, dans toute l'Europe, les facultés de médecine et les salles de dissection avaient le plus grand mal à se fournir en cadavres au point que cette pénurie de corps était devenue un véritable commerce: "Purgé de toute signification symbolique, le cadavre-marchandise est intégré au circuit commercial pour la gloire de la Science et la prospérité des laboratoires» (Debout et Cettour, 2006, p. 107). Au XIX ${ }^{\mathrm{e}}$ siècle, l'alibi scientifique justifie donc toutes les audaces, toutes les transgressions: alors que les fossoyeurs de cadavres se multiplient, l'on constate que la littérature fantastique puise son inspiration dans le réel et lui redonne une dimension symbolique.

Si le corps devient un objet de connaissances, la littérature en fait également un enjeu scientifique et met en scène les salles de dissection ou autres amphithéâtres médicaux comme autant de lieux du fantastique: le château gothique ne fait plus recette et la science toute-puissante, qui avance vers l'inconnu, fournit désormais d'autres motifs de frayeur. Cette évolution du décor fantastique est liée à une nouvelle conception de la mort, qui tend à être désacralisée, comme l'explique Michel Vovelle dans La mort et l'Occident: "La clinique ou l'hôpital - dont la morgue est le complément obligé-prennent place au rang des nouveaux lieux d'une mort dont les traits

AU XIXe SIÈCLE,

L'ALIBI SCIENTIFIQUE JUSTIFIE

DONC TOUTES LES AUDACES,

TOUTES LES TRANSGRESSIONS:

ALORS QUE LES FOSSOYEURS

DE CADAVRES SE MULTIPLIENT,

L'ON CONSTATE QUE

LA LITTÉRATURE FANTASTIQUE

PUISE SON INSPIRATION DANS

LE RÉEL ET LUI REDONNE

UNE DIMENSION SYMBOLIQUE.

ont profondément changé » (Vovelle, 2000, p. 531). Dans Le Rêve du docteur Mišić, la salle d'anatomie devient ainsi le cadre des cauchemars du héros qui se répètent au fil de la nouvelle avec toujours plus d'insistance. C'est dans ce lieu suspendu entre le rêve et la réalité, entre les souvenirs $\mathrm{du}$ docteur et son avenir fatal, qu'apparaît le corps de la jeune bohémienne que le héros désirerait ramener à la vie: «À sa grande stupéfaction une odeur d'acide phénique vint lui chatouiller les narines [...] Il était entré dans une pièce sombre et extrêmement froide, les murs étaient humides et couverts de taches vertes et brunes» (Gjalski, 1996, p. 404-405).
L'odeur de l'acide phénique projette ainsi le docteur Mišić dans une vision inspirée de ses années d'étude, et évoque les anciennes techniques médicales d'anesthésie et de dissection. En effet, Gjalski semble très au fait des substances utilisées à l'époque car le phénol ne fut employé en médecine qu'à partir de 1834. Ainsi, on ne peut réduire la science à un simple arrièreplan car l'auteur l'intègre totalement au récit. En outre, les techniques de dissection sont elles-mêmes évoquées dans l'œuvre car l'autopsie de la jeune bohémienne est décrite avec force détails. C'est à la fin de la nouvelle que se déroule cette scène, annoncée par les nombreux rêves prémonitoires et effrayants du docteur Mišić. Les gestes précis du héros et ses connaissances en anatomie suggèrent davantage un manuel de dissection qu'un simple texte de fiction: "La première entaille faite en croix sur le front lui infligeait une profonde douleur. [...] Lorsqu'il retira la peau du crâne et commença à scier l'os, il fallut qu'il s'arrêtât pour reprendre sa respiration» (Gjalski, 1996, p. 418-419). Avec ces mots crus et ces images choquantes, l'acte médical devient une source d'horreur pour le lecteur; l'auteur utilise ainsi le discours scientifique pour créer un nouvel effet de réel. Pour les besoins de la science, il n'hésite pas à détruire le visage de la jeune femme, dont il vantait la beauté quelques instants auparavant. Désormais écorchée et mutilée par le scalpel du docteur Mišić, elle n'est plus la belle bohémienne mais un cadavre abandonné sur une table d'autopsie.

On peut remarquer que le héros ne parvient pas à déshumaniser totalement le corps de la jeune femme, ni à faire abstraction de sa passion amoureuse. Tel un zombie lui-même, il accomplit l'autopsie avec des gestes mécaniques, suivant un protocole bien établi comme le prouve «l'entaille faite en croix». La marque du scalpel rappelle l'iconographie chrétienne et prend alors une dimension symbolique, comme si cette croix inscrite dans la chair inerte était un présage funeste, annonçant sa propre mort. En oubliant son engagement professionnel et en se laissant aller aux sentiments, le docteur Mišić «signe» son arrêt de mort puisque, par négligence, il s'infligera une entaille qui le conduira à la tombe. Jusqu'à la dernière page, le cadavre de la jeune femme apparaît donc dans la nouvelle et suggère l'obsession grandissante du héros pour la morte. La nécrophilie du personnage est évidente puisque ce corps de femme représente l'objet de ses désirs et de ses soins. La scène de l'autopsie prend même une dimension érotique si l'on analyse la symbolique des gestes du docteur: grâce à la lame du 
scalpel, le héros parvient enfin à accomplir un acte d'amour et à prendre possession de la morte. Il s'abandonne totalement et finit par s'évanouir, à bout de forces, sur le corps de la jeune femme comme s'il expérimentait la «petite mort» de la jouissance. Toutefois, on peut souligner que ce cadavre est presque sacré pour le docteur dans la première partie de l'œuvre, puisqu'il ne songe qu'à le protéger de ses confrères et de la griffe du scalpel: «L'idée que quelqu'un d'autre pourrait examiner le corps lui était insupportable» (Gjalski, 1996, p. 418). C'est sous la contrainte, sous la pression du vieux docteur Asbajer, que le docteur Mišić accepte de pratiquer l'autopsie du corps et de briser le tabou du cadavre.

Dans les œuvres fantastiques, le corps devient donc un spectacle, mis en scène dans les nouveaux "théâtres» que sont les salles de dissection. Ce phénomène, propre à la littérature du XIX $\mathrm{X}^{\mathrm{e}}$ siècle, souligne les transformations profondes de la société et préfigure l'évolution de la télévision et du cinéma de notre époque. Les différentes adaptations de l'œuvre de Mary Shelley permettent de montrer cette évolution et prouvent que le cinéma a mis des décennies avant de représenter ce «spectacle» du corps. En effet, le Frankenstein de James Whale, en 1931, ne dévoile que les pieds des cadavres manipulés par le savant; en 1957, The Curse of Frankenstein de Terence Fisher utilise également les angles de caméra afin de dérober cette réalité aux yeux des spectateurs. Et il faut attendre la fin du $\mathrm{XX}^{\mathrm{e}}$ siècle pour découvrir toute l'horreur des corps décrits en détails dans le roman. L'apparition de ces scènes, toujours plus explicites, n'est pas seulement due à l'évolution des mœurs et s'explique en partie par les progrès techniques réalisés au cinéma. Comme le rappelle l'ouvrage critique de Donald F. Glut, The Frankenstein Archive, les métamorphoses du monstre et des corps qui l'entourent, au fil des décennies, doivent beaucoup au développement des effets spéciaux: "new refinements and details introduced by the make-up artists »(Glut, 2002, p. 8) ${ }^{9}$. L'adaptation de Kenneth Branagh, en 1994, reste en tout cas l'une des plus fidèles au texte-source et n'hésite pas à mettre en scène les cours d'anatomie et de dissection de l'université d'Ingolstadt. Désormais, le réalisateur ne cherche plus à masquer le corps, mais à montrer, au contraire, l'œuvre de la mort. On constate que plus d'un siècle après Mary Shelley, si le cadavre demeure un tabou, il est aussi devenu une source de divertissement. Malgré les formidables progrès de la science, la vie et la création demeurent des zones d'ombre et passion- nent toujours des spectateurs désireux d'être initiés au plus grand des mystères:

Une autre façon d'objectiver ce corps, qui a cours dans des productions filmées plus récentes, est de le transformer en objet d'hyperscience. [...] Là encore le mort est réduit à l'état d'objet, de façon certes plus élaborée: il n'est plus un objet dérisoire mais un objet de science et la déshumanisa-

tion est toujours à l'œuvre (Debout et Cettour, 2006, p. 94).

De nos jours, nombreux sont les programmes télévisés construits autour de la figure du cadavre, et dévoilant de manière impudique l'œuvre sanglante de la mort. Parmi ces émissions spectaculaires, l'on peut citer la série américaine $C S I$ : Crime Scene Investigation (Les Experts, en français) qui connaît un grand succès à travers le monde. Cette série, qui a popularisé les techniques de la police scientifique, accorde une place importante aux autopsies et à l'étude des cadavres: le corps, soumis à toutes sortes d'expériences, devient ainsi une source d'indices nécessaires à la résolution des enquêtes. De même, la série Bones, mettant en scène une anthropologue judiciaire, ouvre pour les spectateurs les portes de la mort. Comme le signale son titre même, l'héroïne tâche de déterminer les causes du décès d'un individu en observant ses os. Dans cette série, le cadavre devient donc un livre codé, accessible aux seuls initiés, et qui doit dévoiler l'histoire d'un homme. Paradoxalement, ce n'est pas l'horreur de la condition humaine qui apparaît dans ce spectacle télévisé, mais l'horreur de l'humanité elle-même dont elle révèle les plus bas instincts, au fil des meurtres toujours plus sanglants. Dans un «monde sans Dieu», le corps est donc devenu un spectacle et semble avoir perdu son caractère sacré.

\section{LE CORPS, ENTRE SCIENCE ET RELIGION}

Dans les sociétés humaines, depuis les plus primitives jusqu'aux plus évoluées, le cadavre a toujours fait l'objet d'un interdit social, moral ou religieux, et représente un tabou. Sigmund Freud propose une définition de ce terme, emprunté au polynésien, dans le chapitre deux de son célèbre ouvrage intitulé Totem et tabou: «Pour nous, le tabou présente deux significations opposées: d'un côté, celle de sacré, consacré; de l'autre, celle d'inquiétant, de dangereux, d'interdit, d'impur» (Freud, 2001, p. 35). L'on retrouve cette ambivalence dans l'image du cadavre qui apparaît à la fois comme une dépouille "sacrée», et comme un corps «impur et dangereux », source de maladies contagieuses et de mystère. C'est pourquoi la question du cadavre est aussi bien religieuse que sociale: son inviolabilité est garantie par les sacrements et les cérémonies qui entourent la mort, mais aussi par la loi. La justice de Dieu et celle des hommes se rejoignent donc autour de cette protection du corps mort, comme le souligne Michel Debout dans Science et mythologie du mort: «[Il] représente ainsi l'interdit que l'homme ne peut atteindre. C'est pour cela que de tous temps le cadavre a été objet de protections, il l'est encore de nos jours, sous la forme du délit qui sanctionne le viol des sépultures » (Debout et Cettour, 2006, p. 62). Mais au XIX ${ }^{\mathrm{e}}$ siècle, la littérature fantastique vient braver cet interdit moral et religieux en s'employant à confondre la vie et la mort. Alors que dans la réalité les règles de la bienséance imposent un rejet de la mort et de ses symboles, le tabou est dépassé par la fiction et marque ce que Philippe Ariès nomme le «décalage entre la mort livresque qui reste bavarde et la mort réelle, honteuse et taisible" (Ariès, 1975, p. 165). Désormais, les cadavres se relèvent de leurs tombes sous forme de vampires ou de morts-vivants, et l'imaginaire fantastique brise peu à peu le tabou entourant ce corps, en nous entraînant dans ce qu'Arlette Boulomié nomme «l'entre-deux-mondes» (Boulomié, 2008, p. 12). Ce phénomène est accentué par les grandes découvertes du siècle scientifique qui poussent les savants à percer les secrets de la nature et du corps humain.

Dans l'Europe du XIX $\mathrm{X}^{\mathrm{e}}$ siècle, au moment où la foi religieuse traverse une crise sans précédent, la science devient une véritable religion et marque la naissance de nouveaux dieux à visage humain : les savants et les médecins. En effet, avec l'essor de la médecine et de la chirurgie, les pouvoirs de ces hommes de science s'étendent et repoussent les interdits :

Cependant le discours médical scien-

tifique a eu, un jour, son mot à dire :

l'unité du corps visible a été brisée,

le corps ouvert, la source de vie

recherchée, l'âme même traquée au

bout du scalpel. Des effets de fantas-

tique nouveaux sont alors devenus

possibles, ce qu'illustre-l'une des premières - Mary Shelley (Bozzetto, 1998, p. 190).

Frankenstein témoigne de cette révolution scientifique qui fait du cadavre l'enjeu d'une lutte de pouvoir entre science et religion. En devenant un objet de connaissances et de recherches sous le scalpel des hommes du XIX ${ }^{\mathrm{e}}$ siècle, le corps mort perd son caractère sacré et cesse ainsi d'être tabou, au premier sens du terme. Dès lors, le cadavre n'est plus considéré comme un simple corps inerte, mais comme un véritable symbole: en parvenant à en 
déchiffrer le sens, le savant est initié aux plus sombres mystères et aux secrets de la Création. Ignorant l'interdit religieux, son œil inquisiteur sonde la chair en décomposition pour découvrir le principe de vie et instaurer sa propre divinisation. Car c'est aux confins des charniers que le docteur Frankenstein cherche des réponses, et c'est grâce aux cadavres qu'il entend créer une nouvelle espèce sur terre: « $A$ new species would bless me as its creator and source; many happy and excellent natures would owe their being to me. No father could claim the gratitude of his child so completely as I should deserve theirs» (Shelley, 1992, p. 55) ${ }^{10}$. Comme le montre cet extrait, le héros est envahi par un sentiment de pouvoir et de démesure, et apparaît davantage comme un dieu que comme un simple créateur. Il devient à cet instant le «Prométhée moderne» qu'évoquait le sous-titre du roman, façonnant un homme de ses mains, non à partir de l'argile mais de matériaux humains. Frankenstein se veut donc une réécriture de la mythologie grecque mais aussi des Saintes Écritures dont s'inspire clairement l'auteur. En effet, comme le Dieu de la Bible, le docteur Frankenstein veut créer une nouvelle race capable de peupler le monde, et voit dans sa créature encore inachevée l'image d'un nouvel Adam qui surpasserait l'homme par sa force et sa stature gigantesque. Mais alors que, dans la Genèse, Dieu s'arrêtait une journée pour contempler son œuvre, le docteur Frankenstein ferme les yeux devant sa créature et ne peut supporter son existence: "the beauty of the dream vanished, and breathless horror and disgust filled my heart. Unable to endure the aspect of the being I had created, I rushed out of the room [...] [I feared] the approach of the demonical corpse to which I had so miserably given life» (Shelley, 1992, p. 58-59) ${ }^{11}$. $\mathrm{Au}$ moment même où la créature monstrueuse voit le jour et ouvre ses grands yeux jaunes, le rêve du savant se brise et le confronte à la dure réalité: il n'est pas un dieu mais simplement un homme, et son nouvel Adam n'est qu'un «cadavre» animé à l'aspect "démoniaque».

La suite du roman de Mary Shelley décrit la lente descente aux enfers du docteur Frankenstein, poursuivi par sa créature vengeresse, et condamné à assister à la destruction de ce qui lui est cher. Il est aisé de voir dans ce dénouement un châtiment destiné à punir l'hybris du héros et son attitude cavalière envers la vie et la mort: son destin tragique serait la conséquence de la violation d'un tabou. Pour avoir ignoré l'interdit entourant le cadavre, le docteur Frankenstein serait donc contraint d'assister à la mort de ses proches, et de voir sa création se changer en destruction. Cette explication nous renvoie à l'article de Northcote $W$. Thomas consacré au «tabou» dans l'Encyclopaedia Britannica (de 1911) et résumé par Sigmund Freud dans Totem et Tabou: «Le châtiment pour la violation d'un tabou était considéré primitivement comme se déclenchant automatiquement, en vertu d'une nécessité interne. Le tabou violé se venge tout seul. Celui qui a violé un tabou est, de ce fait, devenu tabou lui-même» (Freud, 2001, p. 37). Cet article apporte un nouvel éclairage sur le roman dans la mesure où la créature de Frankenstein semble être ce «tabou violé » qui « se venge tout seul». Formée d'un assemblage de membres humains arrachés aux cadavres, cette créature est l'image même de l'interdit; elle incarne la transgression et c'est par elle que la violation sera vengée. En outre, le destin du docteur Frankenstein est très proche de celui du docteur Mišić qui périt dans d'affreuses souffrances à la fin de la nouvelle de Gjalski. Dans cette œuvre, la mort du héros est présentée, une fois encore, comme un juste châtiment sanctionnant la violation de l'interdit. C'est le prix que le docteur Mišić doit payer pour avoir ouvert un cadavre et exploré le corps de la jeune et belle bohémienne qui hantait ses nuits et ses jours. L'auteur souligne l'importance de ce tabou dans la première partie de la nouvelle où l'autopsie de la jeune femme est tout d'abord repoussée comme un sacrilège: "L'homme portait un couteau à la main et se pencha sur la fille pour la disséquer. "Arrête! Elle n'est pas morte! cria-t-il au nouveau venu” »(Gjalski, 1996, p. 405). Dans cet extrait, le cri désespéré du docteur Mišić qui arrête la lame du scalpel illustre parfaitement la puissance du tabou entourant le corps humain. Malgré ses nombreuses connaissances en médecine et son esprit cartésien, le héros ne peut échapper à la peur de l'interdit qui s'exprime dans ce rêve éveillé. Et ce n'est que sous la contrainte que le docteur consent enfin à réaliser l'autopsie à la fin du texte, non sans souffrir dans les tourments de la culpabilité: "Lorsqu'il retira la peau du crâne et commença à scier l'os, il fallut qu'il s'arrêta pour reprendre sa respiration. Il n'arrivait pas à se dominer. Il avait mal et tremblait de tout son corps. [...] Arrivé à la poitrine et aux entrailles, il avait complètement perdu ses forces» (Gjalski, 1996, p. 418-419). Les détails les plus crus de cette intervention suscitent un sentiment d'horreur chez le lecteur et contribuent à l'atmosphère fantastique de la nouvelle.
Comme le docteur Frankenstein, le docteur Mišić subit un châtiment physique pour avoir transgressé le tabou et ignoré le caractère sacré du cadavre. Dans le roman de Mary Shelley, des jours s'écoulent avant que le héros, soutenu par son ami Clerval, puisse enfin retrouver ses esprits et prendre pleinement conscience des conséquences de ses actes. En revanche, dans Le Rêve du docteur Mišić, c'est au moment même où il viole l'interdit que le personnage éprouve de la culpabilité et subit sa punition. En effet, au cours de l'autopsie, le héros de Gjalski s'entaille avec son propre scalpel, ce qui entraîne une infection mortelle; cette fin est symbolique puisque l'on constate que l'arme du chirurgien se retourne contre son propre maître. De plus, l'auteur suggère que le docteur Mišić accepte ce châtiment sans même lutter car il ne signale pas sa blessure à ses collègues et se condamne ainsi à une mort inévitable: «Il accepta et comprit. Sa conception mécaniste et physiologique du monde s'effondrait. Il ne le regrettait pas, au contraire il en ressentait une espèce de plaisir. Emporté par son extase mystique, son âme s'élevait »(Gjalski, 1996, p. 420). Si l'on jette un regard plus scientifique sur ce dénouement, l'on constate que l'auteur s'inspire des découvertes de son époque puisqu'il évoque dans le texte une infection mortelle semblable à l'infection puerpérale qui coûta la vie au savant Ignace Semmelweis. Mais n'oublions pas que la mort du docteur Mišić est symbolique et représente le châtiment d'un homme coupable d'avoir transgressé l'interdit pour les besoins de la science. Dans son article consacré au tabou, N.W. Thomas déclarait, rappelons-le, que "celui qui a violé un tabou est, de ce fait, devenu tabou lui-même» (cité dans Freud, 2001, p. 38). Nulle phrase ne saurait mieux décrire le destin du docteur Mišić contaminé par l'infection mortelle, pour avoir lui-même "violé un tabou». Ainsi, dans l'œuvre de Mary Shelley comme dans celle de Gjalski, c'est dans le châtiment du héros que se révèle le caractère sacré du cadavre.

De tout temps, les hommes ont cherché à assigner une place au cadavre suspendu entre la vie et la mort, qu'il s'agisse du cimetière ou de la salle de dissection. $\mathrm{Au} \mathrm{XIX} \mathrm{X}^{\mathrm{e}}$ siècle, tandis que les sciences ne cessent de progresser, ce cadavre devient un objet de connaissances et se voit élevé au rang de personnage dans la littérature fantastique. Alors que l'homme s'avance désormais dans un monde sans Dieu, alors que «Dieu est mort» selon le mot de Nietzsche, le tabou qui protégeait le corps est remis en question. Sous les yeux des lecteurs, le corps humain est ouvert, 
disséqué, analysé, et devient un enjeu aussi bien scientifique, religieux que social. Cette réflexion autour du cadavre apparaît d'une étonnante modernité car l'on cherche encore de nos jours à déterminer quelle est la place du cadavre dans nos sociétés. Pour preuve, c'est en avril 2009 que l'exposition Our Body présentant au public des cadavres artistiquement mis en scène, a été suspendue dans la ville de Paris, le magistrat chargé de l'affaire ayant décidé que «l'espace assigné par la loi au cadavre est celui du cimetière». Depuis septembre 2010, les écorchés "plastinés » par Gunther von Hagens sont même officiellement interdits sur le sol français, mais continuent leur route dans le monde, depuis l'Allemagne jusqu'aux États-Unis, en laissant derrière eux un parfum de scandale. Si la littérature et la fiction ont le pouvoir de dépasser les interdits, les tabous restent donc puissants dans la réalité et demeurent d'importantes structures sociales.

\section{Bibliographie}

ARIÈS, P. (1975). Essais sur l'histoire de la mort en Occident, Paris, Seuil.

BERTHERAT, B. (2005). «La mort en vitrine à la Morgue à Paris au $\mathrm{XIX}^{\mathrm{e}}$ siècle (1804-1907) », dans R. BERTRAND, A. CAROL et J.-N. PELEN, Les Narrations de la mort, Aix-en-Provence, PUP, coll. «Le Temps de l'histoire», p. 181-195.

BOULOMIÉ, A. (2008). Les vivants et les morts : littératures de l'entre-deux-mondes, Paris, Imago.

BOZZETTO, R. (1998). Territoires des fantastiques: des romans gothiques aux récits d'horreur moderne, Aix-en-Provence, Publications de l'Université de Provence.

CAROL, A. (2004). Les Médecins et la mort, Paris, Aubier.

DEBOUT, M. et D. CETTOUR (2006). Science et mythologie du mort, Paris, Vuibert, coll. «Histoire des sciences».

FREUD, S. (2001). Totem et tabou, Paris, éditions Payot et Rivages.
GAUTIER, T. (2004). «La Morte amoureuse », dans Les mortes amoureuses, Arles, Actes Sud, p. 23-69.

GJALSKI, K.S. (1996 [1890]). «Le Rêve du docteur Mišić», trad. de A. GRCHICH, dans J. GOIMARD et R. STRAGLIATI, $L a$ grande anthologie du fantastique, vol. 1 , Paris, Omnibus, p. 400-421.

GLUT, D.F. (2002). The Frankenstein Archive: Essays on the Monster, the Myth, the Movies, and More, Jefferson, NC et Londres, McFarland and Company Inc.

LECERCLE, J.-J. (1990). "Frankenstein, roman du paradoxe», Les Cahiers des para-littératures, vol. 7: actes du Colloque Frankenstein, Littérature/Cinéma, Liège, éditions C.L.P.C.F., p. 68.

MAURIAC, F. (1970). Journal 1932-1939, Paris, Éditions Bernard Grasset.

PALACIO, J. DE (1969). Mary Shelley dans son ceuvre: contribution aux études shelleyennes, Paris, Klincksieck.

SHELLEY, M. (1975 [1818]). Frankenstein, trad. de G. HANGEST, Paris, GarnierFlammarion.

SHELLEY, M. (1992 [1818]). Frankenstein, Londres, Penguin Classics.

VOVELLE, M. (2000). La mort et l'Occident, de 1300 à nos jours précédé de La mort, état des lieux, Paris, Gallimard.

\section{Notes}

1. Cette nouvelle, qui appartient au recueil Histoires mystérieuses (Tajinstvene priče, 1913), a été écrite en 1890; elle a été traduite en français par Alexandre Grchich et apparaît dans La grande anthologie du fantastique de Jacques Goimard et Roland Stragliati (voir Gjalski, 1996). Le nom de l'écrivain croate Ksaver Šandor Gjalski (1853-1935) est en réalité un pseudonyme pour Lubja Babic.

2. Expression empruntée à l'article de la psychanalyste Laurie Laufer, intitulé "La morgue: voir l'irreprésentable» (<http://recherchespsychanalyse.revues.org/479>).

3. «Après avoir pris cette décision, et passé plusieurs mois à rassembler et disposer convenablement mes matériaux, je commençai mon œuvre» (Shelley, 1975, p. 115).
4. «Dieu, dans sa miséricorde, a fait l'homme beau et attirant, selon sa propre image; mais ma forme n'est qu'un type hideux de la vôtre, rendu plus horrible encore par sa ressemblance même» (Shelley, 1975, p. 210).

5. «Dans une anxiété proche de l'agonie, je rassemblai autour de moi les instruments qui devaient me permettre de faire passer l'étincelle de la vie dans la créature inerte étendue à mes pieds » (Shelley, 1975, p. 119).

6. "Je ramassais des ossements dans les charniers, et mes doigts profanes troublaient les mystères de l'édifice humain. [...] La salle de dissection et l'abattoir me fournissaient une grande partie de mes matériaux » (Shelley, 1975, p. 116).

7. «surgit soudain devant moi la lumière » (Shelley, 1975, p. 113).

8. «Mon attention se fixait sur chacun des objets les plus insupportables pour la délicatesse des sentiments humains. Je voyais la forme magnifique de l'homme s'enlaidir et disparaître; j'observais la corruption de la mort succéder à la fraîcheur des joues vivantes; je voyais le ver prendre pour héritage les merveilles de l'œil et du cerveau »(Shelley, 1975, p. 113).

9. «de nouveaux détails et améliorations introduits par les artistes du maquillage».

10. «Une espèce nouvelle bénirait en moi son créateur et sa source; c'est à moi que devraient l'existence des quantités de nature heureuses et bonnes: nul père ne pourrait mériter la reconnaissance de son enfant comme je mériterais la leur » (Shelley, 1975, p. 115).

11. «la beauté du rêve s'évanouissait, une horreur et un dégoût sans bornes m'emplissaient l'âme. Incapable de supporter la vue de l'être que j'avais créé, je me précipitai hors de la pièce [...] [craignant] l'approche du cadavre démoniaque à qui j'avais donné la vie de façon si misérable» (Shelley, 1975, p. 120). 